\title{
Restoration of spermatogenesis in azoospermic mice by bone marrow mesenchymal stromal/stem cells conditioned medium
}

\section{Rano Zhankina}

Astana Medical University: Astana medical university

\section{Alireza Afshar}

Bushehr University of Medical Sciences

\section{Zohreh Farrar}

Bushehr University of Medical Sciences

Arezoo Khoradmehr

Bushehr University of Medical Sciences

Mandana Baghban

Shiraz University of Medical Sciences

\section{Mahmud Suleiman}

Astana Medical University: Astana medical university

\section{Mohammad Reza Dorvash}

Shiraz University of Medical Sciences

\section{Neda Baghban}

Bushehr University of Medical Sciences

\section{Farhad Rahmanifar}

Shiraz University

Nader Tanideh

Shiraz University of Medical Sciences

Omid Koohi Hosseinabadi

Shiraz University of Medical Sciences

\section{Ali Arabi Monfared}

Shiraz University of Medical Sciences

\section{Shahrokh Zare}

Shiraz University of Medical Sciences

\section{Zahra Khodabandeh}

Shiraz University of Medical Sciences

\section{Mojtaba Farjam}

Fasa University of Medical Science

Iraj Nabipour 
Bushehr University of Medical Sciences

\section{Reza Shirazi}

University of New South Wales Faculty of Medicine

Ulanbek Zhanbyrbekuly

Astana Medical University: Astana medical university

Amin Tamadon ( $\square$ amintamaddon@yahoo.com )

PerciaVista R\&D co. https://orcid.org/0000-0002-0222-3035

\section{Research Article}

Keywords: Azoospermia, Mesenchymal stromal/stem cell, Conditioned media, Bone marrow, Adipose tissue

Posted Date: February 4th, 2022

DOl: https://doi.org/10.21203/rs.3.rs-169243/v2

License: (c) (i) This work is licensed under a Creative Commons Attribution 4.0 International License. Read Full License 


\section{Abstract \\ Background}

One of the main cause of male infertility is non-obstructive azoospermia, which is not manageable medically. The first aim of the current research was to show the effect of extracellular vesicle-contained conditioned media (CM) instead of mesenchymal stromal/stem cells (MSCs) for treatment of nonobstructive azoospermia. In the next step, we aimed to study the differentiation potential of MSCs into spermatocytes after injection of MSCs in mice seminiferous tubules. This study has provided an applied treatment for busulfan-induced azoospermia using adipose tissue-derived (AT-MSCs) and bone marrowderived MSCs (BM-MSCs) and bone marrow CM (BMCM) in animal models.

\section{Methods}

Thirty male adult Balb/C mice ( $30 \pm 5 \mathrm{~g})$ and two female eGFP ${ }^{+/}$Balb/C mice $(30 \pm 5 \mathrm{~g})$ were used to design experimental groups and to culture stem cells, respectively. Then, six groups including intact control, azoospermia, AT-MSC therapy, BM-MSC therapy, BMCM therapy, and spontaneous healing groups were considered. All groups except intact control were induced azoospermia by injecting two doses of busulfan (10 mg/kg) with 21 days' interval. Testes of all mice were removed and studied through histomorphometry and flow cytometry analysis 60 days after treatment.

\section{Results}

Histomorphometry and flow cytometry evaluation of testes showed normal morphology of most of the seminiferous tubules of therapy groups as well as successful recovery of spermatogenesis, but spermatogenesis was not observed in the azoospermia group. It is worth notable that the results of the BM-MSC therapy group were more favorable than other therapy groups.

\section{Conclusions}

AT-MSC, BM-MSC and BMCM can be strongly suggested as candidates in the therapy of azoospermia.

\section{Background}

Infertility is one of the highest serious global severe disabilities that influenced the quality of life of many couples worldwide. In humans, male infertility accounts for $8-12 \%$ of reproductive-aged couples (1), which can be caused by congenital or acquired urogenital abnormalities, malignancies, urogenital tract infections, increased scrotal temperature (e.g. as a consequence of varicocele), endocrine disturbances, genetic abnormalities, immunological factors, and etc. (2). Azoospermia with two types of obstructive or non-obstructive azoospermia affects about $1.3 \%$ of all men (2). This static refers to a notable population 
in the field of male infertility and shows the importance of efforts, which are being made today to treat non-obstructive azoospermia (3).

Although different therapies (for example hormone therapy and testicular sperm extraction) are recommended depending on the type and cause of azoospermia $(4,5)$, about $67 \%$ of azoospermia is related to untreatable testicular disorders, which result in spermatogenic failure (6). Accordingly, researchers have recently tried to develop stem cell transplantation-based therapies for azoospermia (7). These attempts arise from the view that on the one hand, spermatogenic epithelium contains germ cells (spermatogonia) with the ability to repair damages on their own, and on the other hand, if stem cells are placed in testis with defected spermatogonia, they will restore the function of these spermatogonia and induce differentiation/proliferation of them or differentiate to spermatogonia (7).

Various sources of stem cells are used, including embryonic stem cells or pluripotent stem cells, but the use of mesenchymal stromal/stem cells (MSCs) is of great interest as they are free from ethical problems of embryonic stem cells and can be obtained from various sources such as bone marrow, adipose tissue, dental pulp, and menstrual blood, all of which have a high ability to differentiate into different tissues (8). Moreover, MSCs have been shown to secrete cytokines and growth factors that have anti-inflammatory, immune-modulating, anti-apoptotic, and proliferative effects which all are packed in extracellular vesicles (EVs) (9). Exosomes, a nano-scale EV, secreted from cells endosomally that mediate the fusion of endocytic, multicellular vesicles with the plasma membrane of cells (10). They are generally thought to be smaller than other EVs about 30 to $150 \mathrm{~nm}$ in diameter and can be said to be about the size of many lipoproteins but much smaller than cells (11). Exosomes could interact with macromolecules and that they can serve as distributors for proteins, lipids, mRNA, miRNA, and DNA $(12,13)$. They also are capable of being drug carriers since they are composed of cell membranes. Moreover, many studies showed that the exosome could be used as the drug itself due to its anti-inflammatory and immune-modulatory effects (14). In addition, some exosomes from mesenchymal sources showed to have the proliferative effects in vitro and in vivo (15). Conditioned media (CM) of stem cell culture contains the EVs which are secreted from the cells $(16)$. CM can induce tissue regeneration $(17,18)$ or cell differentiation $(19,20)$. In vitro effects of $\mathrm{CM}$ on germ cell regeneration has been shown (20). However, to the best of our knowledge $\mathrm{CM}$ has not been used for the treatment of azoospermia, yet.

On the other hand, bone marrow-derived MSCs (BM-MSCs) and adipose tissue-derived MSCs (AT-MSCs) have recently been considered by many researchers. BM-MSCs and AT-MSCs are used to produce male germ cells in vitro $(21,22)$ and in vivo $(23,24)$. Less invasive, less costly, powerful immunomodulatory effects, more proliferative potential, and more secretion of growth factors and cytokines are the most important priorities of AT-MSCs compared to BM-MSCs for cell therapy (25). As far as we know, there is no comparison between these two sources for cell therapy of azoospermia.

Despite the effectiveness of MSC therapy in the treatment of azoospermia (26), it has yet remained unknown whether transplanted stem cells differentiate to spermatocytes. Based on the existing data, this study is the first attempt to compare the effect of allotransplanting of AT-MSC and BM-MSC and BMCM 
in seminiferous tubules of busulfan induced azoospermia in Balb/C mice. Therefore, the first goal of the present study was to show the effect of EV-contained CM in comparison with MSCs for the treatment of non-obstructive azoospermia. The next aim of this study was to show the differentiation potential of inter-tubular injected MSCs to spermatocytes in mice seminiferous tubules.

\section{Methods}

\section{Animals and ethics}

Thirty male adult Balb/C mice $(30 \pm 5 \mathrm{~g})$ were provided from Research and Clinical Center for Infertility, Shahid Sadoughi University of Medical Sciences, and two female eGFP ${ }^{+/}$Balb/C mice were gifted from Royan Institute and were housed in the laboratory under the controlled condition of a temperature of $22 \pm 2^{\circ} \mathrm{C}$, the humidity of $55 \pm 5 \%$ and lightening cycle of $12 \mathrm{~h}$ light/dark. They were fed ad libitum standard diet. All the manipulations were performed in accordance with the regulations of working with laboratory animals and approved by the Ethical Committee of Astana Medical University, Astana, Kazakhstan (Protocol No. 10).

\section{Isolation and culture of BM-MSC and AT-MSC}

BM-MSCs were isolated from femurs of two eGFP ${ }^{+/}$Balb/C mice by modification of the previously reported method (24). In sterile condition and after soaking the separated thigh of mice in $70 \%$ ethanol, the skin has been removed. After cutting the two ends of the thigh bone, the thigh with the muscles was put in a blue-color sampler tip which its upper half parts had been cut. The tips were placed in $1.5 \mathrm{~mL}$ micro-tubes. Then all micro-tubes were centrifuged at $1500 \times \mathrm{g}$ for $15 \mathrm{~min}$ at $37^{\circ} \mathrm{C}$. The collected $\mathrm{BM}$ at the end of micro-tubes were mixed with $1 \mathrm{~mL}$ of the Dulbecco's modified eagle medium (DMEM) without fetal bovine serum (FBS, Gibco, U.S.A.) and penicillin-streptomycin antibiotics (Gibco, U.S.A.). Then, the BM suspension was transferred to a $25 \mathrm{~cm}^{2}$ flask containing DMEM medium along with $10 \%$ FBS and $1 \%$ penicillin-streptomycin. The flask was incubated at $37^{\circ} \mathrm{C}$, standard humidity, and $5 \% \mathrm{CO}_{2}$ concentration. The culture medium was changed $24 \mathrm{~h}$ after the beginning of incubation, then every $72 \mathrm{~h}$ under sterile conditions, to remove unattached cells and debris. After increasing the density of the cells adhering to the flask to $80 \%$, the cells were passaged using trypsin enzyme. For this purpose, the trypsin enzyme was added to the flask for $3 \mathrm{~min}$ to separate the cells sticking to the flask floor. Then, $2 \mathrm{~mL}$ of $10 \%$ FBS culture medium was poured in to the flask for neutralizing the trypsin enzyme effect. Collecting cells separated from the medium in the first passage was performed and continued till the third passage.

AT-MSCs were provided using ovarian AT of the same mice used in the previous step. Briefly, AT was minced into tiny parts. The AT parts were explanted in a T75 flask. After 15 min of pasting the explants by drying the attachment surface of the explant and flask, they were gently covered with a drop of FBS. The explanted AT were incubated for $48 \mathrm{~h}$ in an incubator containing $5 \% \mathrm{CO}_{2}$ at $37^{\circ} \mathrm{C}$ and saturated humidity. Then DMEM with $10 \%$ FBS and $1 \%$ penicillin and $1 \%$ streptomycin were gently added. By daily monitoring of the border of attachment surface of AT explants and flask, 5 days after explanting and 
observation of AT-MSCs, the explants were removed by tapping the bottom of flask and media replacement. Then, DMEM containing 10\% FBS, 2 mM of L-glutamine (Invitrogen, Netherlands), 1\% penicillin and $1 \%$ streptomycin were added and kept in a $\mathrm{CO}_{2}$ incubator. The sub-culturing of cells was continued till the third passage.

\section{Flow cytometry characterization of MSCs}

To confirm the isolation of BM-MSCs and AT-MSCs and the non-appearance of hematopoietic stem cells, flow cytometry analysis was used. In this regard, the non-appearance of hematopoietic stem cell-specific surface marker expression (CD34 and CD45) and the existence of specific surface area marker expression on BM-MSCs and AT-MSCs (CD44 and CD105) in the isolated cells based on the method previously described were considered (27). Briefly, $5 \times 10^{5}$ cells of the third passage were incubated with specific individual monoclonal antibodies, conjugated with fluorescein isothiocyanate (FITC) or phycoerythrin (PE) in $250 \mathrm{~mL}$ phosphate-buffered saline (PBS) for half an hour at ambient temperature in the dark. CD34, CD 45, CD44, and CD105 primary antibodies were used and cells were diluted using $4 \mathrm{~mL}$ PBS. The diluted cells were centrifuged, and re-suspended in $600 \mathrm{~mL}$ solution containing PBSformaldehyde $2 \%$. Then, they were studied using a four-color FACS Calibur flow cytometer (BD bioscience), and obtained data were analyzed by FlowJo software (FlowJo LLC, Ashland, OR, USA). Immunoglobulin G (IgG)1 FITC and IgG1 PE monoclonal antibodies were applied as isotype controls.

\section{Osteogenic, chondrogenic, and adipogenic characterization of MSCs}

In order to determine the potential of osteogenic, chondrogenic, and adipogenic differentiation of AT-MSC and BM-MSC, the cells of third passage were used. MSCs were in 6-well plates. After fusion, $70 \%$ of the cells were grown in an osteogenic, chondrogenic, or adipogenic environment for 3 weeks. For osteogenic differentiation, the MSCs at $90 \%$ confluence were exposed to an osteogenic differentiation kit (Stem Cell Technology, Iran). Changing the medium was performed two times per week for 3 weeks. After 21 days, osteogenic differentiation was confirmed by Alizarin red staining (Stem Cell Technology, Iran). For chondrogenic induction, MSCs at the confluence of $90 \%$ were exposed to a chondrogenic differentiation kit (Stem Cell Technology, Iran). Induction continued 3 weeks and chondrogenic induction was confirmed by Alcian blue staining (Stem Cell Technology, Iran). For adipogenic induction, MSCs at the confluence of $90 \%$ were exposed to adipogenic differentiation kit (Stem Cell Technology, Iran). Induction continued 3 weeks and induction of adipogenic was confirmed by Oil Red O staining (Stem Cell Technology, Iran). MSCs without differentiation media were stained simultaneously with three protocols as control.

\section{Preparation of BMCM}

In order to obtain the BMCM, BM-MSCs at the third passage were cultured at a density of $10^{6}$ cells in a T75 flask. The BM-MSCs at $80 \%$ confluence were rinsed three times with PBS and $10 \mathrm{~mL}$ of FBS-free DMEM media were replaced. Collection of the media was performed following $48 \mathrm{~h}$ incubation and they were filtrated using a $0.2-\mu \mathrm{m}$ filter to eliminate cellular debris. The BMCM were stored at $-80^{\circ} \mathrm{C}$ until 
injection or isolation and confirmation of EVs. EVs were evaluated by scanning electron microscopy (SEM) or transmission electron microscopy (TEM).

\section{Isolation of EVs of BMCM by kit}

The CM was collected and centrifuged in $2000 \times \mathrm{g}$ and $4^{\circ} \mathrm{C}$ for $30 \mathrm{~min}$. The supernatant was harvested and then added to precipitant reagent of Exovista kit (PerciaVista Co., Iran) with 1:1 ratio. The mixture was kept at $4^{\circ} \mathrm{C}$ for $14 \mathrm{~h}$. To continue, the mixture was centrifuged in $10000 \times \mathrm{g}$ and $4^{\circ} \mathrm{C}$ for $1 \mathrm{~h}$. The EVs' pellet was observed at the bottom of the falcon and collected for further analysis.

\section{SEM imaging of EVs of BMCM}

SEM imaging was performed to show EVs in the isolated BMCM (28). The isolated EVs were fixed with $2 \%$ formaldehyde in PBS. Then, $10 \mu \mathrm{L}$ drop of the sample was placed on poly-l-lysine coated slides and was allowed to air dry for 30-45 min. The sample was washed with phosphate buffered saline (PBS). Then, the sample post-fixed with $2.5 \%$ glutaraldehyde for $30 \mathrm{~min}$. After that, the sample was washed another time for $10 \mathrm{~min}$. Then the sample was stained with $1 \%$ osmium tetroxide for $20 \mathrm{~min}$. The sample was washed again, immediately. At the next step, the sample was dehydrated with accelerated percentage of alcohol $(25,50,70,90,100,100$, and $100 \%$ alcohol). Then the sample was placed into desiccator for drying. The sample was coated with layer of gold. The grid was examined on a FESEM TESCAN MIRA3 machine (TESCAN Co., Czech Republic) operating at resolution of $1.2 \mathrm{~nm}$ at voltage of $30 \mathrm{kV}$.

\section{TEM imaging of EVs of BMCM}

TEM imaging was performed to confirm the presence of EVs in BMCM. The procedure was done based on the previous study (29). In details, $6 \mu \mathrm{L}$ drop of the fixed sample was placed on a formvar Carbon film coated on 200 mesh copper grid (EMS) for 10 min. Excess liquid was absorbed with filter paper. Then, the grid was washed quickly with $100 \mu \mathrm{L}$ MilliQ water and then blotted to remove excess liquid. At the final stage, the grid was placed on $30 \mu \mathrm{L}$ of $1.5 \%$ uranyl acetate (w/v) for $12 \mathrm{~s}$. The grid was examined by PHILIPS CM120 transmission electron microscope (Netherlands) operating at an accelerating voltage of $100 \mathrm{kV}$.

\section{Induction of azoospermia}

In order to model the experimental groups, the male adult Balb/C mice were randomly divided into six equal groups (Figure 1A).

- An intact control group $(n=6)$ : the mice did not receive busulfan and treatment.

- Azoospermia group $(n=6)$ : the left testicles of mice received busulfan injections without treatment and were sampled 60 days following the last busulfan injection.

- BMCM therapy group $(n=6)$ : the right testicles of mice received busulfan injections and then treated with BMCM and were sampled 60 days following the last busulfan injection. 
- BM-MSC therapy group $(n=6)$ : the right testicles of mice received busulfan injections and then treated with BM -MSC and were sampled 60 days following the last busulfan injection.

- AT-MSC therapy group $(n=6)$ : the right testicles of mice received busulfan injections and then treated with AT-MSC and were sampled 60 days following the last busulfan injection.

- Spontaneous healing group $(n=6)$ : the mice received busulfan injections and were left without any treatment and were sampled 120 days following the last busulfan injection.

Then, azoospermia was induced in mice of all groups except those of the intact control group. To induce azoospermia in mice, busulfan was used according to previous reports (24). Briefly, the azoospermia groups were received two doses of busulfan $(10 \mathrm{mg} / \mathrm{kg}$, Busilvex®; Pierre Fabre Medicament Boulogne, France) with 21 days' interval. Thirty-five days following the second injection, their testes were removed and fixed in $10 \%$ formalin buffer solution for histomorphometry studies. The epididymis was collected from all groups and after incubating in PBS, spermatozoa were evaluated by flow cytometry. The testes of control group mice, which were not received busulfan, were assessed through similar procedures.

\section{BM-MSCs and AT-MSCs transplantation}

Preparing an injecting set for a fine cell injection has been previously described (30). The mice in cell and CM therapies' groups ( $n=18$, AT-MSC therapy, BM-MSC therapy, and BMCM therapy groups) were surgically allotransplanted (31). Cell suspensions or CM were mixed with vital stain, sterile trypan blue. The mice were anesthetized with xylazine and ketamine (Alfasan, Netherland) 35 days following the second injection of busulfan. After preparation of abdominal area in dorsal recumbence position, a $1 \mathrm{~cm}$ midline abdominal incision was made to reach the peritoneum and testicle. The fat pad and then right testicle were carefully pulled out using iris forceps under a stereomicroscope (Model SZN, Optika, Italy). Simultaneously, a thin sterile plastic black card with a $30^{\circ} \mathrm{V}$-neck as a holder was placed under the testicle. A polyethylene tube attached to a one-milliliter syringe, was filled with the cell suspension $\left(10^{6}\right.$ cells in $100 \mu \mathrm{L}$ ). The tip of the pipette was carefully inserted into the efferent duct, and slowly threaded a few millimeters toward the testes (Figure 1B). The blue suspension was injected as slowly as possible to avoid moving the pipette and the entrance of the blue suspension into the seminiferous tubules was observed (Figure 1C). Finally, the testes were returned back into the abdomen and the abdominal wall was closed. The untreated testicles on the left side were considered as azoospermia groups.

\section{Histological and histomorphometric assessments}

Sixty days after treatment, the animals' testes were removed after euthanizing with ether. Next, they were put in paraffin for histological and histomorphometric assessments after fixing in 10\% formalin buffer solution and dehydrating using alcohol. For each testis, five horizontal cross-sections with a thickness of $5 \mu \mathrm{m}$ were made from appropriate regions and stained with hematoxylin-eosin for histological assessment. Then, they were studied carefully by means of a light microscope (Model CX21, Olympus, Tokyo, Japan) to evaluate the presence of spermatogonia, spermatocytes, and spermatids in all tubules. Five circular cross-sections were provided from different areas of the tubules and the inner, outer, and total diameters of all the tubules were determined using the Dinocapture software (version 2.0, Dino-Eye, 
San-Chung, Taiwan). Using the mean of two diameters (D1, D2) at right angles, the average diameter of the seminiferous tubules (D) was specified. Using diameter data, cellular (germinal epithelium), luminal, and cross-sectional areas were determined. In the seminiferous tubules, the cross-sectional area (A) was obtained by the equation $A=\pi D 2 / 4$, where $D$ is the average diameter of the tubules, and $\pi$ is 3.142 . The number of seminiferous tubule profiles per unit area was also considered. The area of cells was computed through subtraction of the luminal area from the cross-sectional area. In the seminiferous tubules, determination of the numerical density (Nv) was performed using the following equation:

\section{$N_{V}=N_{A} / D+T$}

where $N_{A}$ indicates the number of profiles per unit area, $D$ displays the average diameter of the seminiferous tubules, T shows the average section thickness, and indicates the number of tubules per unit volume.

The spermatogenesis index including the presence of spermatogenic cells throughout the testicular tissue, affecting the number of cell layers, cell types, and the existence of spermatids in the tubules was studied and the spermatogenic potential of testes was rated based on the modified scale of 0 to 7 (31) as following: 0 , no spermatogonia; 1 , the existence of only spermatogonia; 2 , the presence of spermatocytes; 3 , the presence of spermatids up to 25 in each tubule; 4 , the presence of $25-50$ spermatids per tubule; 5 , the appearance of 50-75 spermatids per tubule; 6 , the appearance of 75-100 spermatids per tubule; and 7 , more than100 spermatids per tubule.

\section{Flow cytometry of enhanced green fluorescent protein expression (eGFP)}

Flow cytometry was conducted to prove the presence of eGFP spermatozoa. For this goal, epididymis was collected from all groups and was incubated in $1 \mathrm{~mL} \mathrm{PBS}$ at $37^{\circ} \mathrm{C}$ for $15 \mathrm{~min}$. Then, they were resuspended in a total volume of $1.8 \mathrm{~mL}$, after adding $800 \mu \mathrm{L}$ of $10 \%$ formalin buffer solution, and then stored on ice until analysis with a flow cytometry under illumination in the range of the $360-400 \mathrm{~nm}$ that is related to eGFP fluorescence (32). A four-color FACS Calibur flow cytometer (BD bioscience) was used to collect the data and data were analyzed using the CellQuest Pro software package (BD bioscience).

\section{Imaging of eGFP spermatozoa}

The imaging of eGFP spermatozoa from the epididymis of treated mice was conducted using an epifluorescent microscope (XDS 3FL4, Optika, Italy) to prove the green fluorescent nature of spermatozoa produced in cell therapy groups. For this purpose, a red filter $(594 \mathrm{~nm})$ and a green filter $(498 \mathrm{~nm})$ were used.

\section{Statistical analysis}

Kolmogorov-Smirnov test was conducted to study the normal distribution of data of histomorphometry indices of seminiferous tubules. All data were presented as means and standard error (mean \pm SE) and 
analyzed using one-way ANOVA and Tukey post-hoc test (SPSS for Windows, version 20, SPSS Inc, Chicago, Illinois, USA). The Mann-Whitney $U$ test was used to assess the spermatogenesis index. A Pvalue of less than 0.05 was considered statistically significant.

\section{Results}

\section{Isolated BM-MSCs and AT-MSCs show stem cells characteristics}

Adherence to plastic and the existence of a homogeneous fibroblast-like population were observed for cultured BM-MSCs and AT-MSCs (Figures 2A and 2B). Additionally, studying the adipogenic, osteogenic, and chondrogenic differentiation abilities of BM-MSCs and AT-MSCs showed the successful differentiation of these cells to adipocytes with intracellular lipid droplets, chondrocytes, and osteoblasts, respectively (Figures $2 \mathrm{C}$ to $2 \mathrm{H}$ ). The diagrams obtained using FlowJo software for characterizing MSCs cell surface markers showed the presence of CD44 and CD105 and the absence of CD34 and CD45 in BM-MSCs and AT-MSCs (Figures $2 \mathrm{I}$ to $2 \mathrm{~L}$ ).

\section{Isolated CM from BM-MSCs contained EVs}

In order to evaluate the quality of CM before injection, EVs visualization with SEM and TEM was performed. We isolated EVs with a commercial kit protocol that allows collecting EVs with a homogeneous diameter size. Furthermore, this protocol removed crystals, protein aggregates, and other residues obtained from $\mathrm{CM}$. The exosomes were detected by SEM and TEM analysis (Figure 2K). The size of exosomes was 20 to $140 \mathrm{~nm}$ in diameter (Figure 2k). In addition, the mean diameter of the exosomes was $74.8 \mathrm{~nm}$. TEM images showed electron-lucent EVs with clear lipid bilayer.

\section{Histologic findings showed therapeutic effects of BMCM, BM-MSCs, and AT-MSCs}

Normal intact mice had condensed germinal epithelium in their seminiferous tubules, and all of the epididymis tubes were filled by spermatozoa (Figure 3A). However, the testes seminiferous tubules of the azoospermia group were empty and their spermatogenesis procedure was completely disrupted (Figure 3B). Seminiferous tubules revealed degenerative variations including germinal epithelia degenerations and seminiferous tubular atrophy in all tubules and no spermatozoa was found in their epididymis. Moreover, thickening of the walls of some epididymal tubes was observed in this group. On the other hand, in the BM-MSCs, AT-MSCs, BMCM, and even the spontaneous healing groups, Sertoli cells, formation of cells of lower layers of germinal epithelium spermatozoa and spermatogonia were observed in some seminiferous tubules (Figures $3 \mathrm{C}$ to $3 \mathrm{~F}$, respectively). Most of the tubules of these groups were nearly filled up with germinal cells including spermatogonia, primary spermatocytes, spermatids, and sperms. Most of the epididymis tubes of this group had spermatozoa, but less than the normal group 
(data not shown). It is evident that between the MSCs therapy groups, the results relating to BM-MSCs therapy were closer to normal intact mice compared to the AT-MSCs therapy group.

\section{Histomorphometry findings indicated the therapeutic effects of BMCM, BM-MSCs and AT-MSCs}

Quantitative histomorphometry as well as qualitative histological analysis must be performed besides the qualitative histological analysis, which can be affected by operator-related bias and does not provide statistically assessable numerical data. Figure 4 indicates the histomorphometric indices of the seminiferous tubules in all studied groups. The cellular area chart shows that intact control, BMCM therapy, AT-MSC therapy, and spontaneous healing group had no significant difference (Figure 4A). Moreover, the maximum and minimum values of this index were related to BM-MSCs therapy and azoospermia groups, respectively. The cross-sectional area chart shows that the maximum and minimum total area of seminiferous tubules were related to the BM-MSC therapy and azoospermia groups (Figure 4B). Control, BMCM therapy, and spontaneous healing groups showed a statistically similar total area. This index had lower values in the ATMSC therapy group than the control group. The graph related to the number of tubules indicates the maximum amount of this index for the azoospermia group (Figure 4C). This index was statistically the same in other groups except for the spontaneous healing group, which showed a slightly higher number of tubule than other groups. The spermatogenesis index of seminiferous tubules of the azoospermia group was minimum (Figure 4D). The control and BMCM therapy groups had no significant difference in terms of this index. ATMSC therapy, BM-MSC therapy, and spontaneous healing groups showed a slightly higher value of this index compared to the control group.

\section{Flow cytometry of eGFP sperms confirmed in vivo differentiation of BM-MSCs and AT-MSCs to spermatozoa}

The results of flow cytometry have been shown in Figure 5. According to this figure, 4.07\%, 61.0\%, 61.5\%, $20.9 \%, 67.4 \%$ and $1.60 \%$ eGFP spermatozoa found in intact control, GFP control, mixed of intact and the eGFP controls, the AT-MSC therapy, the BM-MSC therapy, and the spontaneously healed azoospermia groups, respectively (Figure 5A). The fluorescent-protein-based imaging of spermatozoa produced in cell therapy groups imaging proved the green fluorescent nature of spermatozoa of MSCs therapy groups (Figures 5B to 5E). Spermatozoa were clearly observed in the image obtained by using a green filter (498 $\mathrm{nm})$, while the image taken using a red filter $(594 \mathrm{~nm})$ were not clear. In the flow cytometry analysis, it was expected to observe 0\% eGFP subset in the control group, while this value was $4.07 \%$ that can be considered as noise. In contrast, in the eGFP control group, it was expected to observe $100 \%$ GFP subset, but this value was $61.0 \%$. This difference can be assigned to those sperms whose direction was not appropriate.

\section{Discussion}


The CM and MSC therapies as promising therapy for non-obstructive azoospermia were investigated in mice. Our study for the first time indicated the effects of BMCM for the healing of chemotherapy disrupted the germinal layer in mice. Consistent with our findings, applications of Sertoli cell-CM on the azoospermia model induced spermatogenesis in mice (33). In addition, $10 \%$ of the CM from canine amniotic membrane-derived MSCs enhanced the quality of frozen-thawed sperm cells (34). Since EVs of MSCs contains cell paracrine factors (35), the application of BMCM for azoospermia clinical studies seems to be promising. MSCs-derived CM contains anti-apoptotic factors and growth factors, and it has been investigated in cell-free therapies to simulate the paracrine effects of stem cells on the ovary after chemotherapy (36). Several different cytokines and growth factors with therapeutic properties are secreting from stem cells into their $\mathrm{CM}$, which may play key roles in the regeneration and angiogenesis of damaged tissues (37). Epidermal growth factor (EGF), fibroblast growth factor 2/basic fibroblast growth factor (FGF-2/bFGF), hepatocyte growth factor (HGF), insulin-like growth factor I (IGF-I), insulin-like growth factor II (IGF-II), platelet-derived growth factor (PDGF) and vascular endothelial-derived growth factor (VEGF) are the important growth factors in the CM of MSCs (38). Furthermore, the presence of antioxidants in the $\mathrm{CM}$ (34), in combination with cytokines and growth factors may have an effect on spermatogenesis of azoospermia mice.

Moreover, the findings of our study showed the potential of BM-MSCs and AT-MSCs differentiation to spermatozoa. The flow cytometry graphs related to AT-MSCs therapy and BM-MSCs therapy showed the presence of eGFP sperms indicating the differentiation of transplanted MSCs to sperm. However, the higher percentage of eGFP subset in BM-MSCs therapy group (67.4\%) than AT-MSCs therapy group (20.9\%) revolves higher potential of BM-MSCs to differentiate to sperm compared to AT-MSCs. Additionally, by comparing the graph of the BM-MSCs therapy group with the eGFP control group, it can be concluded that nearly all sperms in the BM-MSCs therapy are GFP+ that also confirms a higher ability of BM-MSCs to differentiate to spermatozoa than AT-MSCs. Germ cell line formation from pluripotent teratocarcinoma cells and successful in vitro generation of offspring mice from embryonic stem cellderived germ cells has been reported for the first time $(39,40)$. The successful differentiation of mice BMMSCs into male germ cells in vitro was also reported (41). The trans-differentiation of BM-MSCs into germ cells, Leydig cells, and Sertoli cells, was shown in busulfan-treated infertile mice $(42,43)$. Human fetal lung- and umbilical cord-derived MSCs were also shown to transdifferentiate into sperm-like cells $(44,45)$. Coherently, recovery of fertility in azoospermia rats following injection of AT-MSCs and their trans-differentiation to spermatozoa have been shown (46). Moreover, the number of tubules except for the azoospermia group with remarkable number of tubules than control and other groups was comparable to the control. The total area and cellular area of all groups except azoospermia, which was remarkably lower than the control group, were comparable with the control group, confirming the successfulness of studied therapy in treating azoospermia mice. Moreover, it is notable that higher values of these indexes for the BM-MSCs therapy group in comparison to others demonstrate more significant effectiveness of this therapy method over other studies in the current work. However, induction of spermatogenesis with AT-MSCs and BM-MSCs has been shown in various animal models $(23,24,31,46-$ 
49), although these two sources were compared by our findings for the first time and the priority of BMMSCs for this purpose was shown.

\section{Conclusions}

Spermatogenesis was effectively induced in seminiferous tubules of busulfan-induced azoospermic mice through allotransplantation of BMCM, AT-MSCs, and BM-MSCs. BMCM and its related EVs could induce recovery of the germinal layer in a model of non-obstructive azoospermia. However, the attained results using the BM-MSCs therapy was slightly more effective than AT-MSCs and BMCM therapies. The results of histomorphometric and flow cytometry analysis have been clearly demonstrated that the recovery of spermatogenesis using MSCs occurs through two parallel mechanisms. First, repairing defected spermatogonia and second, differentiation to spermatogonia as a result of the effect of their $\mathrm{CM}$. These golden achievements provide a platform for the clinical study of CM therapy of azoospermia in men.

\section{Abbreviations}

AT-MSCs, adipose tissue-derived mesenchymal stromal/stem cells

BM-MSCs, bone marrow-derived mesenchymal stromal/stem cells

$\mathrm{CM}$, Conditioned media

$D$, average diameter of the seminiferous tubules

DMEM, Dulbecco's modified eagle medium

EGF, Epidermal growth factor

eGFP, fluorescent protein expression

EVs, extracellular vesicles

FBS, fetal bovine serum

FGF-2/bFGF, fibroblast growth factor 2/basic fibroblast growth factor

FITC, fluorescein isothiocyanate

HGF, hepatocyte growth factor

IGF-I, insulin-like growth factor I

IGF-II, insulin-like growth factor II

MSCs, mesenchymal stromal/stem cells 
$N_{A}$, number of profiles per unit area

$N_{V}$, numerical density

PBS, phosphate buffered saline

PDGF, platelet-derived growth factor

$\mathrm{PE}$, phycoerythrin

SE, standard error

SEM, scanning electron microscopy

$T$, average section thickness

TEM, transmission electron microscopy

VEGF, vascular endothelial-derived growth factor

\section{Declarations}

\section{Ethics approval and consent to participate}

All the manipulations were performed in accordance with the regulations of working with laboratory animals and approved by the Ethical Committee of Astana Medical University, Astana, Kazakhstan (Protocol No. 10).

\section{Consent for publication}

Not applicable.

\section{Availability of data and material}

Data are available as requested.

\section{Funding}

This study was partly funded by a grant from the Department of Urology and Andrology, Astana Medical University, Astana, Kazakhstan by Dr. Ulanbek Zhanbyrbekuly (Grant no. AP08857153) and Fasa University of Medical Sciences by Dr. Mojtaba Farjam. Some part of this study has been financially supported by The Council for Stem Cell Sciences and Technologies, affiliated to the Iranian Vice 
Presidency for Science and Technology for the development of exosome isolation kit by Dr. Amin Tamadon.

\section{Competing interests}

Author Amin Tamadon is employed by PerciaVista R\&D Co. The remaining authors declare that the research was conducted in the absence of any commercial or financial relationships that could be construed as a potential conflict of interest.

\section{Authors' contributions}

A.T., U.Z., R.S., M.F. and I.N. conceived and designed the format of the manuscript. R.Z., A.A., Z.F., A.K., M.B., M.S., M.R.D., N.B., F.R., N.T., O.K.H., A.A.M., S.Z. and Z.K. collected data, analysed the findings, drafted and edited the manuscript. A.T., U.Z., R.S., M.F. and I.N. reviewed the manuscript. All authors contributed to the critical reading and discussion of the manuscript. All authors have read and agreed to the published version of the manuscript.

\section{Acknowledgments}

The authors would like to thank the Stem Cells Technology Research Center for their collaboration with laboratories. The authors wish to thank Dr. Yaser Tahamtani from Royan Institute for his kindly supports.

\section{References}

1. Duca Y, Calogero AE, Cannarella R, Condorelli RA, La Vignera S. Current and emerging medical therapeutic agents for idiopathic male infertility. Expert Opin Pharmacother. 2019;20(1):55-67.

2. Punab M, Poolamets O, Paju P, Vihljajev V, Pomm K, Ladva R, et al. Causes of male infertility: a 9-year prospective monocentre study on 1737 patients with reduced total sperm counts. Hum Reprod. 2017;32(1):18-31.

3. Zhang D, Liu X, Peng J, He D, Lin T, Zhu J, et al. Potential spermatogenesis recovery with bone marrow mesenchymal stem cells in an azoospermic rat model. Int J Mol Sci. 2014;15(8):13151-65.

4. Tharakan T, Salonia A, Corona G, Dhillo W, Minhas S, Jayasena C. The Role of Hormone Stimulation in Men With Nonobstructive Azoospermia Undergoing Surgical Sperm Retrieval. J Clin Endocrinol Metab. 2020;105(12):dgaa556.

5. Shin T, Kobayashi T, Shimomura Y, Iwahata T, Suzuki K, Tanaka T, et al. Microdissection testicular sperm extraction in Japanese patients with persistent azoospermia after chemotherapy. Int J Clin Oncol. 2016;21(6):1167-71. 
6. Esteves SC. Clinical management of infertile men with nonobstructive azoospermia. Asian J Androl. 2015;17(3):459-70.

7. Vij SC, Sabanegh E Jr, Agarwal A. Biological therapy for non-obstructive azoospermia. Expert Opin Biol Ther. 2018;18(1):19-23.

8. Shirazi R, Zarnani AH, Soleimani M, Nayernia K, Ragerdi Kashani I. Differentiation of bone marrowderived stage-specific embryonic antigen 1 positive pluripotent stem cells into male germ cells. Microscopy research technique. 2017;80(4):430-40.

9. Galipeau J, Sensebe L. Mesenchymal Stromal Cells: Clinical Challenges and Therapeutic Opportunities. Cell Stem Cell. 2018;22(6):824-33.

10. van Niel G, D'Angelo G, Raposo G. Shedding light on the cell biology of extracellular vesicles. Nat Rev Mol Cell Biol. 2018;19(4):213-28.

11. Edgar JR. Q\&A: What are exosomes, exactly? BMC Biol. 2016;14(1):1-7.

12. Naseri Z, Oskuee RK, Jaafari MR, Forouzandeh Moghadam M. Exosome-mediated delivery of functionally active miRNA-142-3p inhibitor reduces tumorigenicity of breast cancer in vitro and in vivo. Int J Nanomedicine. 2018;13:7727-47.

13. Raposo G, Nijman HW, Stoorvogel W, Liejendekker R, Harding CV, Melief CJ, et al. B lymphocytes secrete antigen-presenting vesicles. J Exp Med. 1996;183(3):1161-72.

14. Fang W, Vangsness CT. Jr. Implications of Anti-Inflammatory Nature of Exosomes in Knee Arthritis. Cartilage. 2020:1947603520904766.

15. Zhang S, Chuah SJ, Lai RC, Hui JHP, Lim SK, Toh WS. MSC exosomes mediate cartilage repair by enhancing proliferation, attenuating apoptosis and modulating immune reactivity. Biomaterials. 2018;156:16-27.

16. Park KS, Bandeira E, Shelke GV, Lasser C, Lotvall J. Enhancement of therapeutic potential of mesenchymal stem cell-derived extracellular vesicles. Stem Cell Res Ther. 2019;10(1):288.

17. Almeria $C$, Weiss R, Roy M, Tripisciano $C$, Kasper $C$, Weber $V$, et al. Hypoxia conditioned mesenchymal stem cell-derived extracellular vesicles induce increased vascular tube formation in vitro. Front Bioeng Biotechnol. 2019;7:292.

18. Bazoobandi S, Tanideh N, Rahmanifar F, Zare S, Koohi-Hosseinabadi O, Razeghian-Jahromi I, et al. Preventive Effects of Intrauterine Injection of Bone Marrow-Derived Mesenchymal Stromal CellConditioned Media on Uterine Fibrosis Immediately after Endometrial Curettage in Rabbit. Stem Cells Int. 2020;2020:8849537.

19. Jahromi IR, Mehrabani D, Mohammadi A, Seno MM, Dianatpour M, Zare S, et al. Emergence of signs of neural cells after exposure of bone marrow-derived mesenchymal stem cells to fetal brain extract. Iran J Basic Med Sci. 2017;20(3):301-7.

20. Shah SM, Saini N, Singh MK, Manik R, Singla SK, Palta P, et al. Testicular cell-conditioned medium supports embryonic stem cell differentiation toward germ lineage and to spermatocyte-and oocytelike cells. Theriogenology. 2016;86(3):715-29. 
21. Luo Y, Xie L, Mohsin A, Ahmed W, Xu C, Peng Y, et al. Efficient generation of male germ-like cells derived during co-culturing of adipose-derived mesenchymal stem cells with Sertoli cells under retinoic acid and testosterone induction. Stem Cell Res Ther. 2019;10(1):91.

22. Ghasemzadeh-Hasankolaei M, Eslaminejad MB, Sedighi-Gilani M. Derivation of male germ cells from ram bone marrow mesenchymal stem cells by three different methods and evaluation of their fate after transplantation into the testis. In Vitro Cellular Developmental Biology-Animal. 2016;52(1):4961.

23. Karimaghai N, Tamadon A, Rahmanifar F, Mehrabani D, Raayat Jahromi A, Zare S, et al. Spermatogenesis after transplantation of adipose tissue-derived mesenchymal stem cells in busulfan-induced azoospermic hamster. Iran J Basic Med Sci. 2018;21(7):660-7.

24. Tamadon A, Mehrabani D, Rahmanifar F, Jahromi AR, Panahi M, Zare S, et al. Induction of Spermatogenesis by Bone Marrow-derived Mesenchymal Stem Cells in Busulfan-induced Azoospermia in Hamster. Int J Stem Cells. 2015;8(2):134-45.

25. Xu L, Liu Y, Sun Y, Wang B, Xiong Y, Lin W, et al. Tissue source determines the differentiation potentials of mesenchymal stem cells: a comparative study of human mesenchymal stem cells from bone marrow and adipose tissue. Stem Cell Res Ther. 2017;8(1):275.

26. Zhanbyrbekuly U, Tamadon A, Akhnazarov SK, Suleiman M, Aynayev E, Zhaparov U, et al. Using mesenchymal stem cells for the treatment of non-obstructive azoospermia. Urologiia (Moscow, Russia: 1999). 2020(4):119-23.

27. Ghaneialvar H, Soltani L, Rahmani HR, Lotfi AS, Soleimani M. Characterization and classification of mesenchymal stem cells in several species using surface markers for cell therapy purposes. Indian $\mathrm{J}$ Clin Biochem. 2018;33(1):46-52.

28. Reclusa P, Verstraelen P, Taverna S, Gunasekaran M, Pucci M, Pintelon I, et al. Improving extracellular vesicles visualization: From static to motion. Sci Rep. 2020;10(1):6494.

29. Rikkert LG, Nieuwland R, Terstappen L, Coumans F. Quality of extracellular vesicle images by transmission electron microscopy is operator and protocol dependent. Journal of extracellular vesicles. 2019;8(1):1555419.

30. Kasraeian M, Ghasemi E, Dianatpour M, Tanideh N, Razeghian IJ, Khodabandeh Z, et al. In utero xenotransplantation of mice bone marrow-derived stromal/stem cells into fetal rat liver: An experimental study. International Journal of Reproductive BioMedicine. 2020;18(9):701.

31. Rahmanifar F, Tamadon A, Mehrabani D, Zare S, Abasi S, Keshavarz S, et al. Histomorphometric evaluation of treatment of rat azoospermic seminiferous tubules by allotransplantation of bone marrow-derived mesenchymal stem cells. Iranian Journal of Basic Medical Sciences. 2016;19(6):653.

32. Balestrini PA, Jabloñski M, Schiavi-Ehrenhaus LJ, Marín-Briggiler Cl, Sánchez-Cárdenas C, Darszon A, et al. Seeing is believing: Current methods to observe sperm acrosomal exocytosis in real time. Molecular Reproduction and Development. 2020. 
33. Panahi S, Karamian A, Sajadi E, Aliaghaei A, Nazarian H, Abdi S, et al. Sertoli cell-conditioned medium restores spermatogenesis in azoospermic mouse testis. Cell Tissue Res. 2020;379(3):57787.

34. Mahiddine FY, Kim JW, Qamar AY, Ra JC, Kim SH, Jung EJ, et al. Conditioned Medium from Canine Amniotic Membrane-Derived Mesenchymal Stem Cells Improved Dog Sperm Post-Thaw QualityRelated Parameters. Animals (Basel). 2020;10(10):1899.

35. Yuan QI Z, Yg, Chen Q. Mesenchymal Stem Cell (MSC)-Derived Extracellular Vesicles: Potential Therapeutics as MSC Trophic Mediators in Regenerative Medicine. Anat Rec. 2020;303(6):1735-42.

36. Hong L, Yan L, Xin Z, Hao J, Liu W, Wang S, et al. Protective effects of human umbilical cord mesenchymal stem cell-derived conditioned medium on ovarian damage. J Mol Cell Biol. 2020;12(5):372-85.

37. Gama KB, Santos DS, Evangelista AF, Silva DN, de Alcântara AC, dos Santos RR, et al. Conditioned medium of bone marrow-derived mesenchymal stromal cells as a therapeutic approach to neuropathic pain: a preclinical evaluation. Stem Cells Int. 2018;2018.

38. Noverina R, Widowati W, Ayuningtyas W, Kurniawan D, Afifah E, Laksmitawati DR, et al. Growth factors profile in conditioned medium human adipose tissue-derived mesenchymal stem cells (CMhATMSCs). Clinical Nutrition Experimental. 2019;24:34-44.

39. Nayernia K, Li M, Jaroszynski L, Khusainov R, Wulf G, Schwandt I, et al. Stem cell based therapeutical approach of male infertility by teratocarcinoma derived germ cells. Hum Mol Genet. 2004;13(14):1451-60.

40. Nayernia K, Nolte J, Michelmann HW, Lee JH, Rathsack K, Drusenheimer N, et al. In vitrodifferentiated embryonic stem cells give rise to male gametes that can generate offspring mice. Dev Cell. 2006;11(1):125-32.

41. Nayernia K, Lee JH, Drusenheimer N, Nolte J, Wulf G, Dressel R, et al. Derivation of male germ cells from bone marrow stem cells. Lab Invest. 2006;86(7):654-63.

42. Drusenheimer N, Wulf G, Nolte J, Lee JH, Dev A, Dressel R, et al. Putative human male germ cells from bone marrow stem cells. Soc Reprod Fertil Suppl. 2007;63:69-76.

43. Lue Y, Erkkila K, Liu PY, Ma K, Wang C, Hikim AS, et al. Fate of bone marrow stem cells transplanted into the testis: potential implication for men with testicular failure. Am J Pathol. 2007;170(3):899908.

44. Kim J, Seandel M, Falciatori I, Wen D, Rafii S. CD34+ testicular stromal cells support long-term expansion of embryonic and adult stem and progenitor cells. Stem Cells. 2008;26(10):2516-22.

45. Hua J, Qiu P, Zhu H, Cao H, Wang F, Li W. Multipotent mesenchymal stem cells (MSCs) from human umbilical cord: potential differentiation of germ cells. Afr J Biochem Res. 2011;5(4):113-23.

46. Cakici C, Buyrukcu B, Duruksu G, Haliloglu AH, Aksoy A, Isik A, et al. Recovery of fertility in azoospermia rats after injection of adipose-tissue-derived mesenchymal stem cells: the sperm generation. Biomed Res Int. 2013;2013:529589. 
47. Mehrabani D, Hassanshahi MA, Tamadon A, Zare S, Keshavarz S, Rahmanifar F, et al. Adipose tissuederived mesenchymal stem cells repair germinal cells of seminiferous tubules of busulfan-induced azoospermic rats. J Hum Reprod Sci. 2015;8(2):103-10.

48. Hajihoseini M, Vahdati A, Ebrahim Hosseini S, Mehrabani D, Tamadon A. Induction of spermatogenesis after stem cell therapy of azoospermic guinea pigs. Vet Arh. 2017;87(3):333-50.

49. Hajihoseini M, Mehrabani D, Vahdati A, Hosseini SE, Tamadon A, Dianatpour M, et al. Spermatogenesis after Transplantation of Adipose Tissue-Derived Stem Cells in Azoospermic Guinea Pigs: A Histological and Histomorphometric Study. Galen Medical Journal. 2018;7:1000.

\section{Figures}



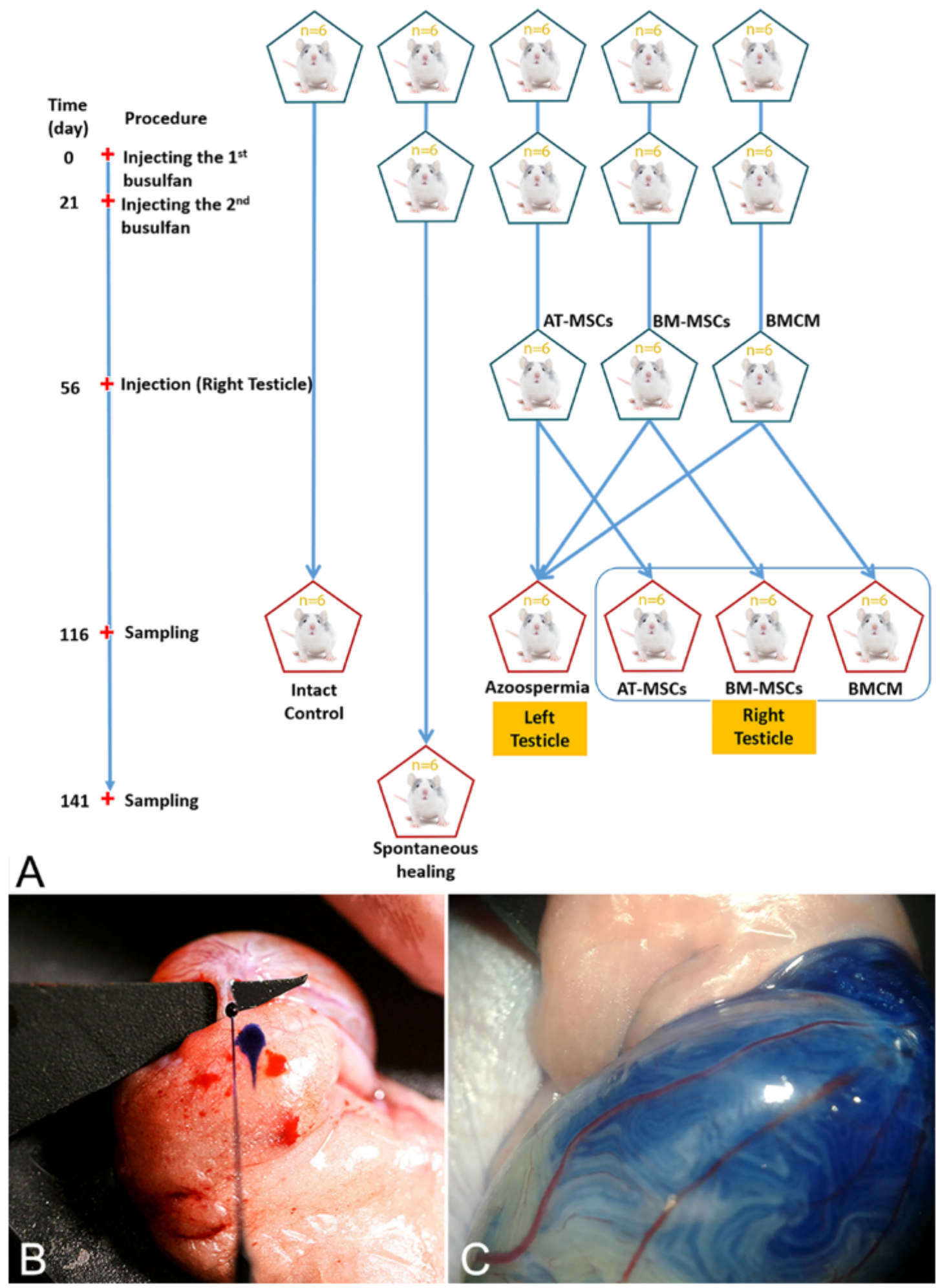

Figure 1

Procedures of inter-tubular injection of bone marrow-derived mesenchymal stromal/stem cells (BMMSCs), adipose tissue-derived MSCs (AT-MSCs), and BM conditioned medium (BMCM). A) Schematic procedures of experiments to evaluate the restoration of spermatogenesis in azoospermic mice by bone marrow-derived mesenchymal stromal/stem cells (BM-MSCs), BM conditioned medium (BMCM), and adipose tissue-derived MSCs (AT-MSCs). B) A triangular hard black plastic card was inserted underneath 
the efferent duct. C) Therapeutic cells or BMCM were mixed with trypan blue were injected into an efferent duct of mice testis.

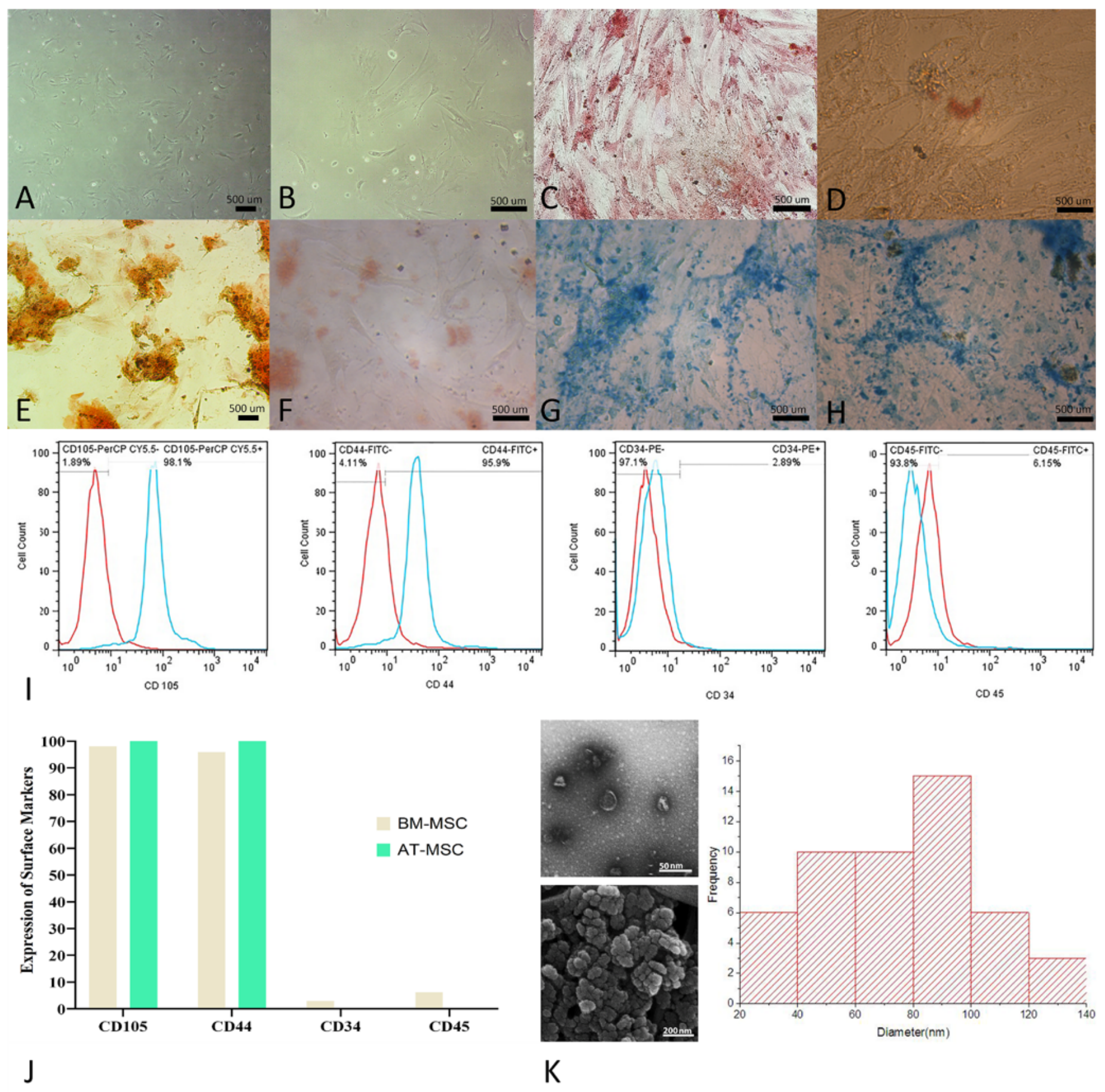

Figure 2

Confirmation of mesenchymal stromal/stem cells (MSCs) and extracellular vesicles (EVs) from bone marrow-derived MSCs conditioned medium (BMCM) isolations. Adherence cells to plastic and morphology: A) BM-MSCs, B) adipose tissue-derived MSCs (AT-MSCs). Adipogenic differentiation: C) BM- 
MSCs, D) AT-MSCs; Osteogenic differentiation: E) BM-MSCs, F) AT-MSCs; chondrogenic differentiation: G) BM-MSCs, H) AT-MSCs. Phenotype by flow cytometry analysis: I) Expression of BM-MSCs surface markers (CD44 and CD105) (>95\%) in contrast to negative expression hematopoietic markers (CD34, CD45) $(<10 \%) \mathrm{J}$ ) Expression of AT-MSCs surface markers (CD44 and CD105) (>95\%) in contrast to negative expression hematopoietic markers (CD34, CD45) $(<10 \%)$. K) Confirmation of isolation of EVs with scanning electron microscopy (SEM), transmission electron microscopy (TEM) and frequency of EVs diameters. 


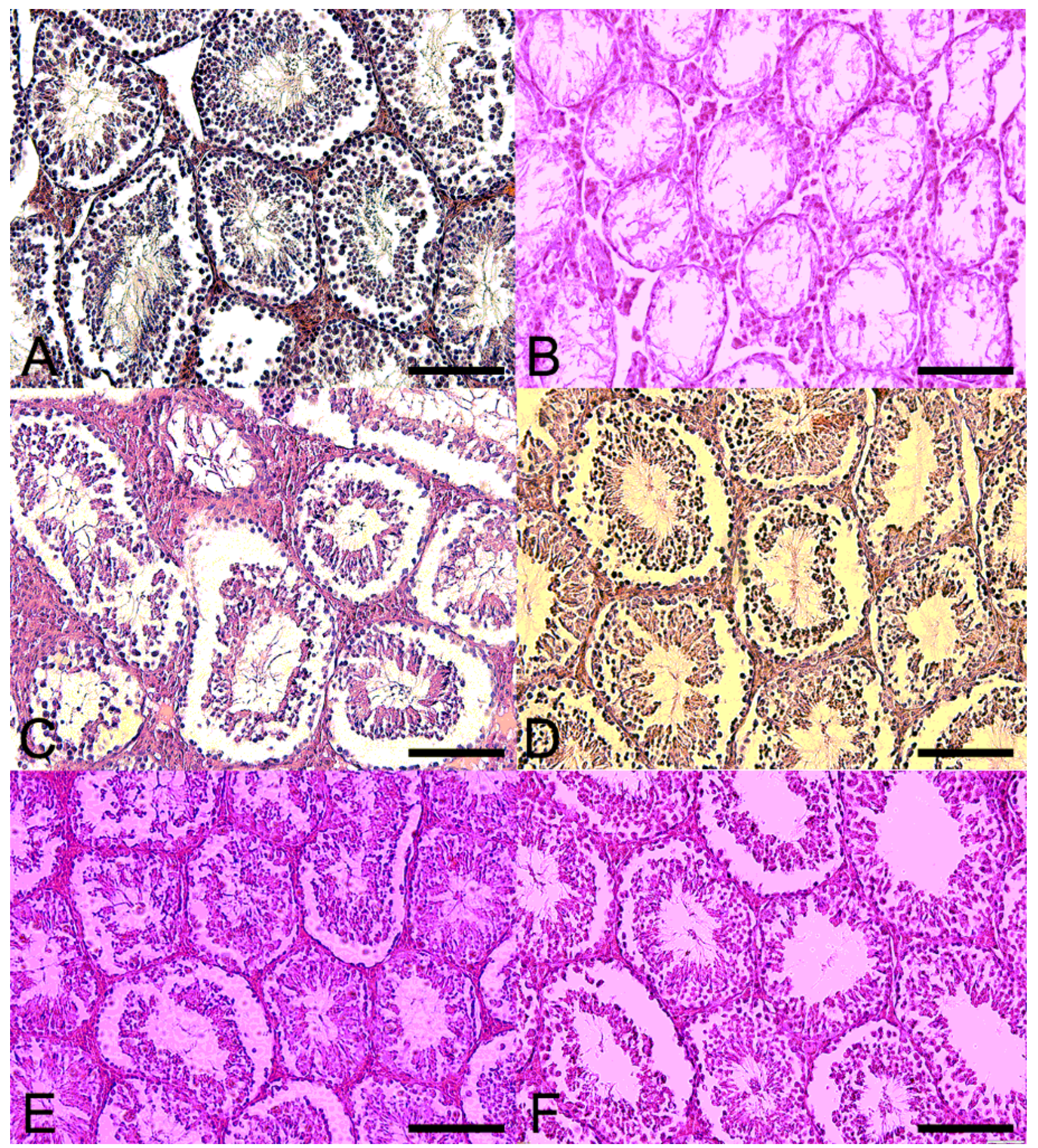

Figure 3

Histologic comparison of restoration of spermatogenesis in azoospermic mice by bone marrow-derived mesenchymal stromal/stem cells (BM-MSCs), adipose tissue-derived MSCs (AT-MSCs), and BM conditioned medium (BMCM). A) Seminiferous tubules of normal control mice with condensed spermatogenic epithelium. B) Seminiferous tubules of busulfan-treated azoospermic mice were partially empty and without germinal layer cells indicating the absence of spermatogenesis. C) Seminiferous 
tubules of treated mice with BM-MSCs. Some of the tubules appeared to start spermatogenesis but not completely repaired. D) Seminiferous tubules of treated mice with AT-MSCs show the presence of germinal epithelial cells and tubes are filled up with spermatogenic cells. E) Seminiferous tubules of treated mice with BMCM indicate spermatogenesis and some tubes are filled up with spermatozoa. F) Spontaneous healing of busulfan treated azoospermic mice 120 days after chemotherapy. Hematoxylin and eosin staining and all scale bars are $40 \mu \mathrm{m}$.
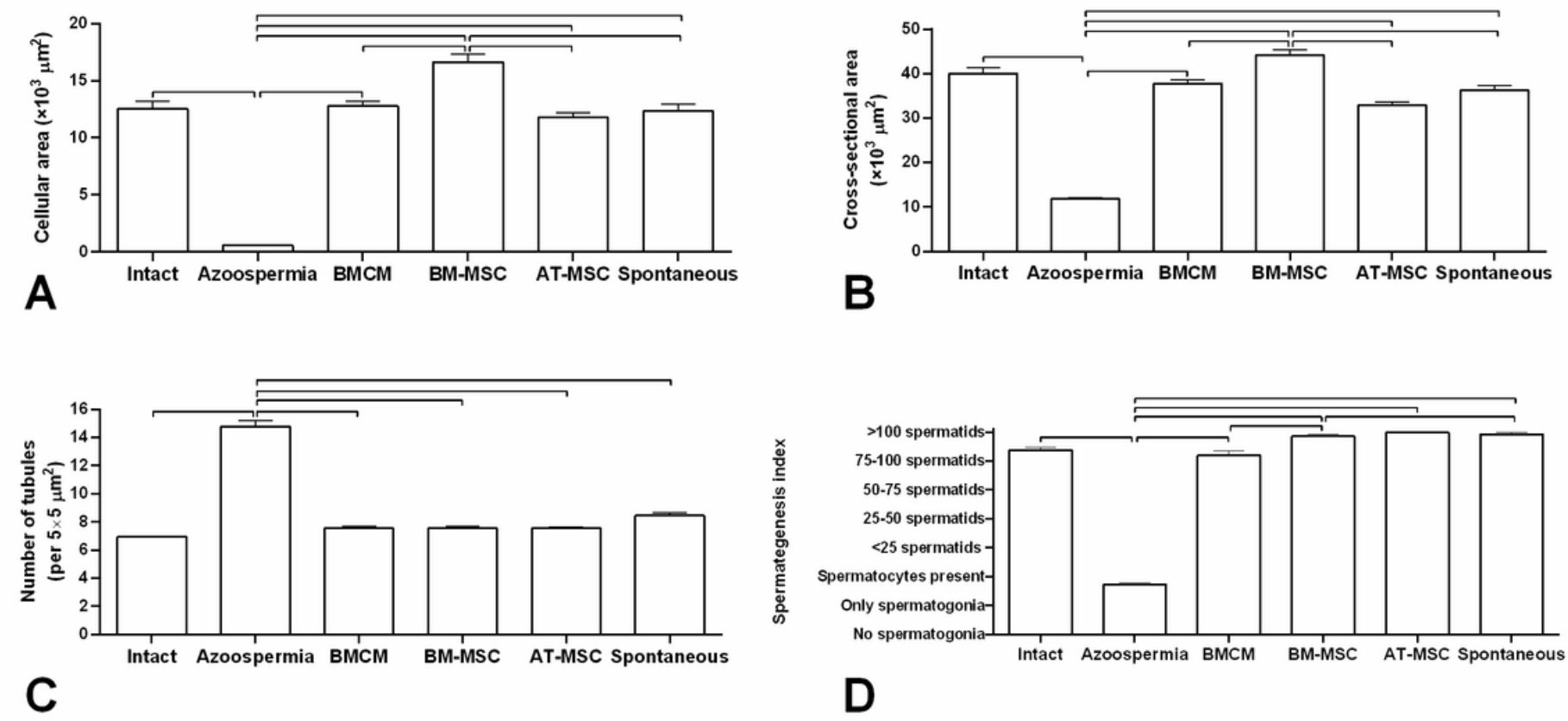

\section{Figure 4}

Mean and standard error of histomorphometric indices of seminiferous tubules in busulfan induced azoospermic testis of mice treated with bone marrow-derived mesenchymal stromal/stem cells (BMMSCs), adipose tissue-derived MSCs (AT-MSCs), and BM conditioned medium (BMCM) in comparison with intact control, azoospermic, and spontaneous healing groups. A) cellular area, B) cross-sectional area of the tubule, C) number of tubules per $5 \times 5 \mathrm{~mm}^{2}$, D) spermatogenesis index of seminiferous tubules. Lines above the columns show significant differences between groups $(P<0.05)$. 

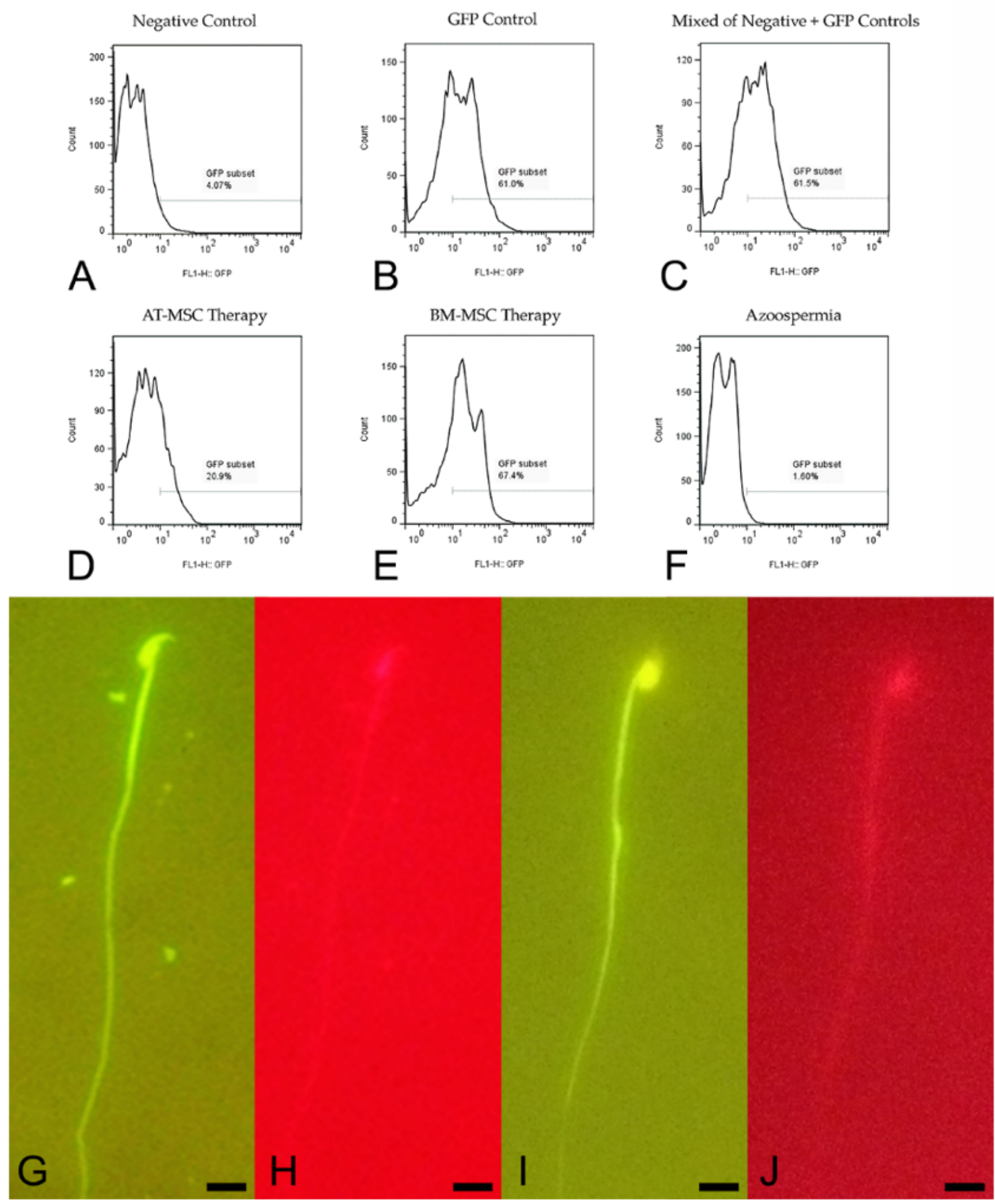

\section{Figure 5}

Confirmation of production of eGFP spermatozoa in bone marrow-derived mesenchymal stromal/stem cells (BM-MSCs) and adipose tissue-derived MSCs (AT-MSCs) treated azoospermic mice. Flow cytometry of expression of enhanced green fluorescent protein (eGFP) in spermatozoa of A) negative control mice, B) eGFP positive mice, C) combination of spermatozoa of negative control mice $(0.5 \mathrm{~mL})$ and eGFP positive mice $(0.5 \mathrm{~mL}), \mathrm{D})$ azoospermic mice treated with AT-MSCs eGFP $\left.{ }^{+}, \mathrm{E}\right)$ azoospermic mice treated 
with BM-MSCs eGFP ${ }^{+}, \mathrm{F}$ ) azoospermic mice that was spontaneously healed. Imaging of eGFP ${ }^{+}$ spermatozoa of azoospermic treated mice with BM-MSCs eGFP ${ }^{+}$in $\mathrm{G}$ ) green filter and $\mathrm{H}$ ) red filter and ATMSCs eGFP ${ }^{+}$in I) green filter and $\mathrm{J}$ ) red filter. All scale bars are $10 \mu \mathrm{m}$. 